\title{
Virtual conferencing and meeting systems \\ Resources for online connections
}

$\mathbf{L}$ ibrarians have always tried to stretch the limits of applicable technologies to create effective connections among researchers and resources. We thrive on the tools and tactics that help us do this, and we are always on the prowl for newer, better alternatives. This is one of the best ways we have of keeping high service standards in the face of shrinking budgets and skeletal staffing. Fortunately, there have never been so many technologies to choose from, and there have never been so many challenges calling out for technological intervention.

Hosting online instruction sessions is, for example, an excellent opportunity for libraries to benefit from new technologies. As the private sector has known for some time, good virtual conferencing systems can save time and money by reducing the need for travel, and they provide surprising flexibility for online training. Facing increasing demands to provide library-related instruction for classes held off-campus and online, we undertook a search for the best products to serve academic needs and budgets.

What follows are the results of our investigation: a small selection of some of the most popular, the most cost-effective, and the most useful systems available. This list is by no means complete, however. It seems that there are new entries to the market almost every day, each with its own strengths. We hope the entries below will help you get started on your own search for the best of the many alternatives.

\section{Comprehensive systems}

These are among the best-known, most widely used virtual meeting and conferencing systems available. They boast a full selection of features, and most can be customized with institutional logos and colors.

- Adobe Connect. Adobe Connect is a highly flexible, comprehensive virtual meeting system. It requires no client downloads and works with nearly any electronic device. Customization options are almost limitless, and branding for your organization is easy. Presentation spaces can be saved and reused, or a customized URL can be set for persistent, instant access to meeting rooms. Meetings can be recorded, reused, and distributed. Nearly any type of file can be stored and used for presentations: documents, audio, video, animation, presentation software, and others. A variety of learning assessments may be used from within the online classroom, including quizzes, simulations, and engagement monitoring. Educational and institutional pricing is available. Access: http://www.adobe.com/products /adobeconnect.edu.html.

John Gottfired is coordinator of reference services, email: john.gottfried@wku.edu, Laura DeLancey is coordinator of electronic resources, email: laura.delancey@wku.edu, Carol Watwood is health sciences librarian, email: carol. watwood@wku.edu, and Amanda Hardin is reference services specialist, email: amanda.hardin@wku.edu, at Western Kentucky University Libraries

๑ 2015 John Gottfried, Laura DeLancey, Carol Watwood, and Amanda Hardin 
- Blackboard Collaborate. Blackboard Collaborate is a highly versatile application that's useful for virtual meetings and online presentations. The user is able to organize a virtual meeting room in which participants can interact at any time. The attendees can enter using a specific web link or from the Blackboard website. Blackboard Collaborate also features an interactive whiteboard, the ability to create separate rooms for chats, and multipoint video. Both single and multiuse licenses are available. The presenter can record the session and share with other users at a later time. Attendees can participate using either a microphone or a telephone connection. A one-time software download is required, but no plugins or updates are needed, and the software is compatible with all popular browsers and platforms. Mobile apps are available for both Android and iOS. Access: http://www.blackboard.com /platforms/collaborate/overview.aspx.

- Citrix GoToMeeting. Citrix GoToMeeting is an easy-to-set-up, flexible web conferencing product appropriate for any meeting, presentation, and webinar where visual elements are needed. Setup is minimal, requiring only an online launcher. The individual version allows up to 25 participants. Invitations are sent by URL. For Windows users, Outlook integration is available. Participants enter meetings using an ID number, while an audio pin enables calling in by phone. Chat support and a 24/7 toll-free helpline are available. A "WebViewer" function allows visitors to view sessions via a web browser, if the launcher fails to execute. Videos files may be shared via social media or saved onto a computer in a variety of formats. Online drawing tools are available. Additional features such as polling are available on the premium GoToWebinar and GoToTraining products. Business and organizational pricing is available. Access: www.gotomeeting.com/.

- Microsoft Lync. Microsoft Lync is a full-featured conferencing and communication program. It offers smooth integration with Microsoft applications and Skype. Mobile apps are available for iOS, Android, and Windows, along with a web-based app usable from most common web browsers. Among the most popular features are easy one-touch connections to online meetings and instant connection to Microsoft email and calendar systems. Access: http://products.office.com/en/lync/.

- WebEx. WebEx offers a comprehensive virtual meeting application providing simultaneous screen sharing and live video streams, polling options, whiteboard, chat, and document sharing. Sessions can be recorded for distribution and re-use, and both live and recorded sessions are accessible via URL and access code. WebEx is available as an app for Apple, Android, Windows, and Blackberry mobile devices, while desktops require users to download a plugin. WebEx has both free and paid options ranging from three participants to services appropriate for large-scale webinars or online courses, including quizzes and discussion forums. Access: http://www.webex.com.

\section{Free systems}

Many virtual conferencing products offer a basic level of free access, but free access is fundamental to the design and marketing plan of the following systems.

- Google Hangouts. Google Hangouts is a free (for up to ten participants) voice, chat, and video calling service. For full functionality on a desktop, a Google+ account is needed. Google Hangouts is quick and easy to set up-desktop users need to create a Google account, which takes minutes. Stand-alone mobile apps are available for Android and iOS (Apple). Calls to the United States and Canada are free. Videos and photos may be shared; additional apps enable polling, whiteboard, and other special functions. For the young at heart, a distinctive feature is the large available library of colorful emoji emoticons. Google Hangouts on Air allows sharing of videos to a large audience using YouTube; sessions are automatically saved and uploaded. Editions for business, government, and education have additional features for which Google+ is not needed. Access: https://plus.google.com/hangouts. 
- Skype. Skype is an intuitive video and voice calling platform, available as an app or download for any mobile device or computer. It is compatible with some televisions and gaming consoles, and there is a beta browser version (Skype for Web). The desktop version allows up to ten users to participate simultaneously, and each can share their screen, chat, send pictures and documents, and talk using camera and microphone. Skype to Skype calls are free, though both users must have a Skype account. Free calls are limited to four hours per call, ten hours per day, and 100 hours per month. Access: http://www. skype.com/en/.

\section{Quick and easy meetings}

The applications below offer quick, nearly effortless meeting startups, useful for popup instruction requests or fast meetings.

- Join.Me. Join.Me is a simple collaborative system designed to help users create online meetings quickly and intuitively. Available as an app or desktop download, Join.Me offers a very basic free system serving up to ten participants, with paid options allowing up to 250. It boasts low costs with the added benefit of unlimited audio. Instead of a link sent through the program or email, Join.Me generates a nine-digit code to share with each participant. The system allows file transfers and flexible screen-sharing (allowing participants to control the host's screen for interactivity and demonstrations). Join. Me updates via plugins and does require certain browsers and Flash Player 10 to run efficiently. Access: https://www.join.me/en.

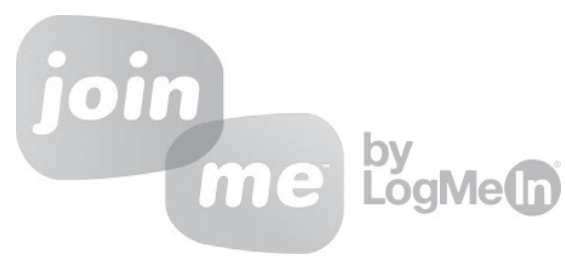

- MeetingBurner. This is a strong system for meetings and instruction. The cloud-based format does not require a software download, and it is compatible with most desktops and mobile applications. MeetingBurner makes it extremely easy to set up a quick meeting or demonstration, and you can change hosts instantaneously. It allows the use of screen-sharing, streaming video of the host, chat messaging, and audio communication (computer or phone). Number of participants permitted varies by account type, from ten with free access up to 1,000 with the Premier account. Meetings can be started immediately or scheduled for the future, and promoted via internal connection to Facebook, or through a registration widget that can be mounted on any web page. Access: https://www. meetingburner.com.

\section{Also worth considering}

- ClickMeeting. ClickMeeting is a cloudbased system through which voice or visual meetings and conferences can be held using any common browser. It allows screensharing and online chat (both individual and moderated group chat). ClickMeeting allows you to brand conferences with your organization's logos, colors, and graphics. The base level allows up to 25 participants per session, with higher levels offering up to 1,000. Costs are reasonable, and customized pricing arrangements are available. Access: http://www.clickmeeting.com/.

- Fuze.com. Fuze.com (formerly FuzeBox) allows HD video conferencing on most mobile and desktop devices. The system offers screen-sharing, whiteboard, and PowerPoint presentations, and it works with Dropbox and Box files. Fuze permits scheduling through Outlook or Google Calendar. The free version permits only three participants, but other pay plans are available. It requires installation of software (for desktop) or app (for mobile devices). Set-up is easy, and the system is generally easy to operate. Access: https://www.fuze.com/.

- GlobalMeet. PGI's GlobalMeet has a clean, intuitive interface and is available on 
most mobile and desktop devices. It allows customizable URLs, and guests are not required to download any software or plugins. All plans include screen sharing, whiteboard, chat, polls, and meeting recording, and there are both pay-as-you-go and subscription options. Access: https://www.globalmeet.com/.

- ilinc. There are separate modules designed specifically for meetings, classroom learning, and webinars. This system provides both video and audio conferencing with up to 25 participants in meetings, up to 50 for classrooms, and up to 1,000 for webinars. The system offers a full range of services, including screen-sharing, file distribution, whiteboard systems, chat, and others. The session presenter can monitor audience engagement, monitor feedback, and allow participants to take over as host of the session, permitting full interactivity. The iLinc system is compatible with both Mac and PC systems. Access: http://www.ilinc.com/.

- MegaMeeting. This is a browser-based web and video conferencing product using Adobe Flash; no download is required. User joins meetings by email or direct link, and password access is available. It works across multiple platforms and devices. MegaMeeting provides whiteboard and polling, and it allows file-sharing, including video, documents, and others. The basic "webinar" version allows one live video stream per meeting; "Professional" service allows 16. The number of seats depends on the option selected; the purchased number of seats may be broken up into multiple simultaneous "rooms." Recording is optional. Branding and other customized features are offered, and educational discount pricing is available. MegaMeeting provides live customer support 24/7. Access: www.megameeting.com/.

- ReadyTalk. This is an audio and web conferencing service in which the host installs a one-time download. Participants attend meetings online, accessing with a seven-digit code. Invitations may be sent by Facebook and Twitter. Files can be loaded and shared during meetings. Cross-platform and mobile access is available. Features include prepa- ration mode, polling, chat, drawing tools, recording, and post-meeting follow-up. Help is available by chat or $24 / 7$ helpline. The basic monthly plan allows 25 participants; multiple pricing options are offered to accommodate additional users. Access: http:// www.readytalk.com/.

- UberConference. UberConference is available as a Chrome extension, desktop app, or Google or Apple mobile app. It offers screen-sharing and conference calls, and integrates well with social media profiles (Facebook, LinkedIn, and Google +), as well as Dropbox, Google Drive, and EverNote for document sharing. Conference calls do not require a PIN to participate. Free and paid options are available. Access: https://www. uberconference.com.

- Yugma. This conferencing system works on most desktops (Windows, Mac, and Linux), and it integrates with Skype for video conferencing. Yugma allows screen sharing, document sharing up to $100 \mathrm{MB}$, and online chat (individual and group). Sessions can be recorded for later viewing and sharing. Various pricing plans permit up to 500 participants. The system does require a software download, and it requires the use of Java. Access: https://yugma.com/.

- WebInterpoint. Onstream Media's Web Interpoint conferencing tools integrate with Microsoft PowerPoint, Word, Excel, and Outlook. The system allows file transfers, polling, and chat. The host can record sessions, and it has a customizable interface that permits institutional branding. Pricing is based on usage. Access: http://www.onstreammedia. com/webinterpoint.php. $\boldsymbol{n}$

\section{C\&RL News RSS}

Cover art, article links, and other valuable information from CERL News is available by subscribing to our RSS feed.

Point your Web browser to feeds. feedburner.com/candrlnews, and add our feed to your favorite feed reader. 